\title{
Synthesizing Chaotic Maps with Prescribed Invariant Densities
}

\author{
Alan Rogers ${ }^{\mathrm{a}, *}$ Robert Shorten ${ }^{\mathrm{b}}$ Daniel M. Heffernan ${ }^{\mathrm{c}, 1}$

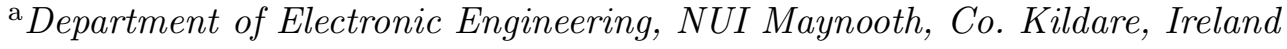 \\ ${ }^{\mathrm{b}}$ Hamilton Institute, NUI Maynooth, Co. Kildare, Ireland \\ ${ }^{\mathrm{c}}$ Department of Mathematical Physics, NUI Maynooth, Co. Kildare, Ireland
}

\begin{abstract}
The Inverse Frobenius-Perron problem (IFPP) concerns the creation of discrete chaotic mappings with arbitrary invariant densities. In this note, we present a new and elegant solution to the IFPP, based on positive matrix theory. Our method allows chaotic maps with arbitrary piecewise-constant invariant densities, and with arbitrary mixing properties, to be synthesized.
\end{abstract}

Key words: Chaos, Chaotic Maps, Chaos Control, Inverse Frobenius-Perron Problem

PACS: 05.45.-a, 02.50.-r

\section{Introduction}

The synthesis, or custom-design of chaotic maps, is a natural extension of the research carried out on nonlinear dynamical systems over the past thirty years. Ulam and Von Neumann had even studied the logistic map in the 1940s [1]. Notwithstanding Von Neumann's famous point about using deterministic processes to generate randomness, synthetic chaotic maps do have many potential applications, especially in hardware-based random-number generators, and digital noise generators [2-4]. They may also be used to artifically recreate physical data from real-world systems [5]. In this paper, we present an elegant

* Corresponding Author.

Email address: alan.rogers@eeng.may.ie (Alan Rogers).

1 Also at: School of Theoretical Physics, Dublin Institute for Advanced Studies, Dublin 4, Ireland 
way of creating designer chaotic maps with arbitrary invariant densities. The synthesis method presented is based on the theory of positive matrices, and originates in work on synchronised communication networks.

When a chaotic map is iterated, different initial conditions exponentially diverge leading to completely different long-run behaviour. However, when looked at statistically, many chaotic maps possess a single physically relevant invariant density, which remains stable when random noise is added to the process [6]. Given an arbitrary initial condition, the invariant density describes where iterates end up on average. For simple maps such as the tent map, or the Bernoulli $(2 x \bmod 1)$ map, iterates have an equal probability of landing anywhere in the state-space: the natural invariant density $\rho$ is constant, and equals one [7]. Many maps, however, do not possess a simple invariant measure.

The Frobenius-Perron operator $P_{\tau}$ is an operator on the space of probability density functions [8]. The invariant density is a fixed point of the FrobeniusPerron equation:

$$
P_{\tau} f(x)=\frac{d}{d x} \int_{\tau^{-1}[a, x]} f d \lambda
$$

While it is relatively straightforward to calculate the invariant density $\rho$ of piecewise affine maps, most continuous maps do not possess a closed-form solution to the Frobenius-Perron equation (especially where the invariant density is a fractal).

Clearly, if it is very difficult to analyse a map to find its invariant density, then the synthesis problem - generating a map which possesses a desired invariant density - must seem quite a daunting prospect. However, Ulam conjectured that the Frobenius-Perron operator could be approximated by the action of a Markov map acting on a partition of the interval concerned [9]. The Ulam conjecture was proved in 1976 by Li [10]. The Ulam matrix is a column stochastic matrix which gives the probability of moving from any particular interval in the partition to any other interval. The principle eigenvector of the Ulam matrix is the invariant density of the map. Thus if a Markov matrix can be synthesized for a particular eigenvector, then we have a solution for the Inverse Frobenius-Perron problem (IFPP). The IFPP has been tackled and solved by various groups (see [11] and references therein). The method most relevant to this work is the solution of Góra and Boyarsky [12], [5]. They show how to generate a 3-band transformation from any given invariant density. However, our method is more direct in that both the eigenvector and the Ulam transition matrix are parameterized. No work is required to generate the matrix - the procedure is completely mechanical. Our method also gives complete control over the mixing properties of the map, independent of the invariant density. 
In the following section, we outline the synthesis method. We then give a simple example of the application of the method to synthesize a map with a prescribed invariant density. Some properties of the transition matrix are also given, followed by conclusions. The appendix gives a little extra background on the origins of the matrix used.

\section{Synthesis Method}

The following matrix arises naturally in the dynamical analysis of synchronised communication networks based on TCP (Transmission Control Protocol). Further properties of the matrix are given in the appendix, and the interested reader should refer to [13], or [14], for the origins of this matrix.

$$
A=\left(\begin{array}{cccc}
\beta_{1} & 0 & \cdots & 0 \\
0 & \beta_{2} & 0 & 0 \\
\vdots & 0 & \ddots & 0 \\
0 & 0 & \cdots & \beta_{n}
\end{array}\right)+\frac{1}{\sum_{i=1}^{n} \alpha_{i}}\left(\begin{array}{c}
\alpha_{1} \\
\alpha_{2} \\
\vdots \\
\alpha_{n}
\end{array}\right)\left(1-\beta_{1} 1-\beta_{2} \cdots 1-\beta_{n}\right)
$$

The matrix $A$ is a column stochastic matrix, and is strictly positive when $\alpha_{i} \geq 0$ and $0<\beta_{i}<1 \quad \forall i \in\{1, \cdots, n\}$, and so $A$ can represent a Markov process. From the theory of positive matrices, it is well-known that the matrix $A$ has a leading eigenvalue $\rho(A)=1$, and a single eigenvector in the positive orthant, called the Perron eigenvector [15]. The Perron eigenvector, $x_{p}$ of the $A$ matrix has the following form:

$$
x_{p}^{T}=\left(\frac{\alpha_{1}}{1-\beta_{1}}, \ldots, \frac{\alpha_{n}}{1-\beta_{n}}\right)
$$

Clearly, we can control the dominant eigenvector of the matrix through choice of the $\alpha_{i}$ and $\beta_{i}$. For our purposes, the matrix $A$ is the Ulam matrix, and the Perron eigenvector represents the invariant density of the process governed by $A$. The Ulam matrix may be represented as a one-dimensional chaotic map if we let each entry of the matrix denote the transition probability from one interval to another. More formally, partition the unit interval into $N$ equal subintervals, $\left\{I_{1}, \ldots, I_{N}\right\}$. Let entry $a_{j i}$ of the $A$ matrix denote the probability of a transition from interval $I_{i}$ to interval $I_{j}$, denoted $p_{i j}$. To construct the map, place a line segment of slope $\pm 1 / p_{i j}$ in the square defined by the intervals $I_{i}, I_{j}$, for each entry of the matrix. It is convenient to start at the point $(0,0)$ and place the line segments end to end, although there are many possibilities, as illustrated in Figure 1. The map in Figure 1 is one possible implementation of the following transition matrix: 


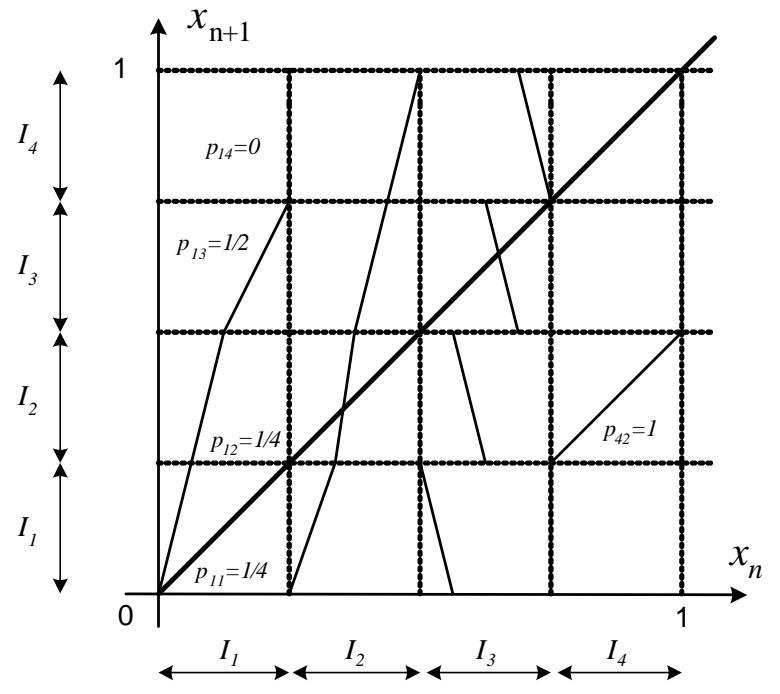

Fig. 1. A possible one-dimensional map corresponding to a $4 \times 4$ transition matrix

$$
A=\left(\begin{array}{cccc}
1 / 4 & 1 / 3 & 1 / 4 & 0 \\
1 / 4 & 1 / 6 & 1 / 4 & 1 \\
1 / 2 & 1 / 4 & 1 / 4 & 0 \\
0 & 1 / 4 & 1 / 4 & 0
\end{array}\right)
$$

We can write the matrix $A$ in the following, more compact, form, where we have normalized the alphas: $\sum \alpha_{i}=1$.

$$
A=\left(\begin{array}{cccc}
\beta_{1}+\alpha_{1}\left(1-\beta_{1}\right) & \alpha_{1}\left(1-\beta_{2}\right) & \cdots & \alpha_{1}\left(1-\beta_{n}\right) \\
\alpha_{2}\left(1-\beta_{1}\right) & \beta_{2}+\alpha_{2}\left(1-\beta_{2}\right) & & \\
\vdots & & \ddots & \\
\alpha_{n}\left(1-\beta_{1}\right) & & & \beta_{n}+\alpha_{n}\left(1-\beta_{n}\right)
\end{array}\right)
$$

It is possible to choose the $\alpha_{i}$ and $\beta_{i}$ so that the eigenvector represents any desired invariant density. For simplicity, we can set all of the $\beta_{i}=0.1$ say, and then determine the $\alpha_{i}$ for the desired density. Once the $\alpha_{i}$ and $\beta_{i}$ are determined, the matrix $A$ is fully determined, and can then be implemented as a map. 


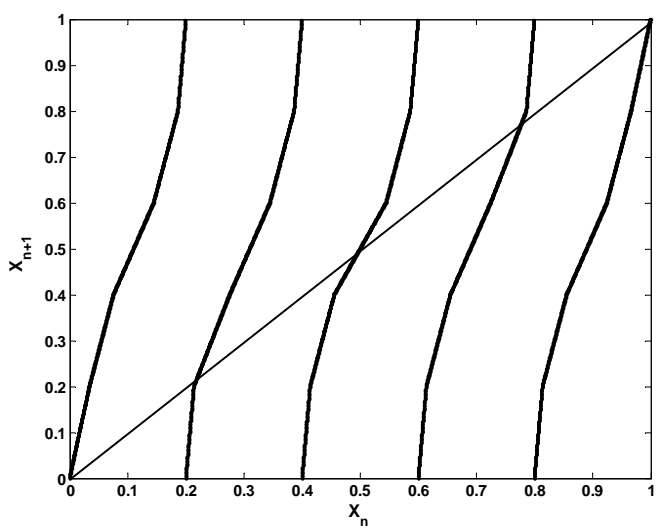

Fig. 2. Map corresponding to the A matrix in the example (6)

\section{Example}

As an example, we will synthesize a chaotic map whose invariant density has a triangular shape: For simplicity, we partition the unit interval into five equal segments. Let $\rho_{\text {desired }}=[1,3,5,3,1]$. We then determine the $\alpha_{i}$ with $\beta_{i}=0.1$. This gives us the following column-stochastic transition matrix:

$$
A=\left(\begin{array}{lllll}
0.1692 & 0.0692 & 0.0692 & 0.0692 & 0.0692 \\
0.2077 & 0.3077 & 0.2077 & 0.2077 & 0.2077 \\
0.3462 & 0.4462 & 0.3462 & 0.3462 & 0.3462 \\
0.2077 & 0.2077 & 0.2077 & 0.3077 & 0.2077 \\
0.0692 & 0.0692 & 0.0692 & 0.0692 & 0.1692
\end{array}\right)
$$

A possible 1-D chaotic map corresponding to this transition matrix is shown in Figure 2, and the invariant density of the map after 30000 iterations, $\rho_{\text {actual }}$ is shown in Figure 3. The density has been scaled such that the first entry of $\rho_{\text {actual }}=1$ to allow ready comparison with the desired invariant density. It can be seen that $\rho_{\text {actual }}$ is very close to the desired invariant density, $\rho=[1,3,5,3,1]$. Figure 4 shows a typical output from the map. (MATLAB code to implement the method is freely available at http://www.eeng.may.ie / arogers/ifpp.) 


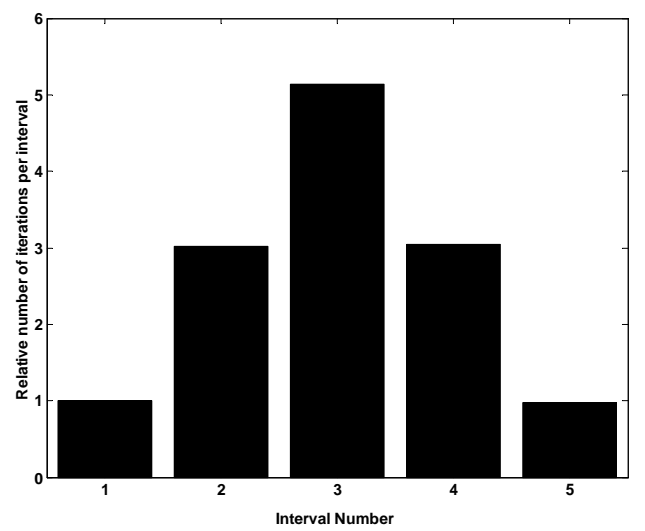

Fig. 3. Invariant density of map in Figure 2 after 30000 iterations

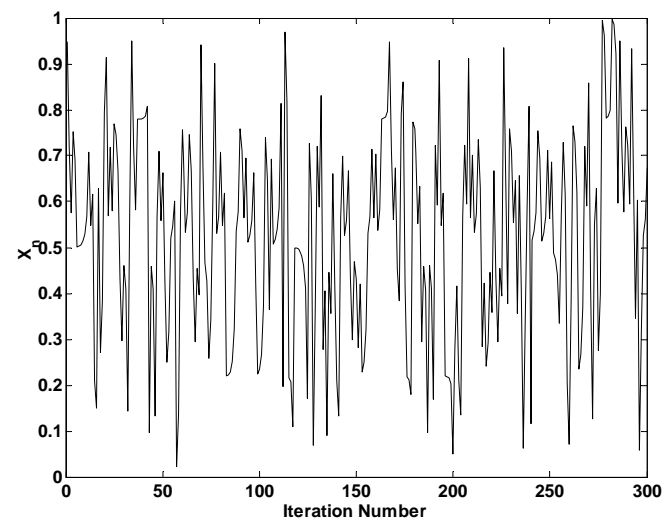

Fig. 4. Chaotic time-series produced by the map in Figure 2

\section{Further Properties of the Transition Matrix}

The matrix $A$ arises naturally in the study of synchronised communication networks, and is used to model the dynamics of networks employing TCP congestion control [13] [14]. It has a number of interesting properties, which we mention here.

(1) The matrix $A$ is strictly positive as defined in section 2 , or strictly nonnegative if we allow $\alpha_{i}=0$. As such, it possesses a dominant eigenvalue called the Perron eigenvalue $\lambda_{p}$ of geometric and algebraic multiplicity 1 . The corresponding Perron eigenvector

$$
x_{p}^{T}=\left(\frac{\alpha_{1}}{1-\beta_{1}}, \ldots, \frac{\alpha_{n}}{1-\beta_{n}}\right)
$$

represents a fixed point of the linear system $W(k+1)=A W(k)$. For any other eigenvalue $\lambda_{i} \neq \lambda_{p}$, we have $\left|\lambda_{i}\right|<\lambda_{p}$. (2) The rate of convergence to the fixed point is bounded by the second 
largest eigenvalue of $A$. For our application, the second-largest eigenvalue determines how quickly we get to the desired invariant density. The second-largest eigenvalue thus controls the mixing properties of the map. As we can make the second-largest eignevalue as large or as small as we like, we have complete control over the mixing properties, independent of the invariant density. It can be shown that, apart from the Perron eigenvalue, all the the eigenvalues of $A$ lie within the interval $\left[\beta_{1}, \beta_{n}\right]$.

(3) If the $\beta_{i}$ are distinct, and are ordered such that $\beta_{1}<\beta_{2}<\cdots<\beta_{n}$, then it can be shown that the eigenvalues of $A$ are interlaced with the $\beta_{i}$ thus:

$$
\beta_{1}<\lambda_{1}<\beta_{2}<\lambda_{2}<\cdots<\beta_{n}<\lambda_{n}=\lambda_{p}=1
$$

(4) The Lyapunov exponent of the map resulting from the matrix $A$ can be shown to be

$$
\Lambda \simeq \alpha_{1} \alpha_{2} \ldots \alpha_{n} \ln \frac{1}{\alpha_{1} \alpha_{2} \ldots \alpha_{n}}+\alpha_{1}^{2} \ln \frac{1}{\alpha_{1}}+\cdots+\alpha_{n}^{2} \ln \frac{1}{\alpha_{n}}
$$

in the limit $\beta_{i} \rightarrow 0$. We find this to be a very good approximation for small $\beta_{i}$.

\section{Conclusions}

From an engineering viewpoint, custom-design of chaotic maps is absolutely necessary for possible future applications. Maps must be tailored to fit the application. In this paper, we have presented a direct way of creating designer chaotic maps with arbitrary invariant densities. The synthesis method presented is based on the theory of positive matrices, and originated in work on synchronised communication networks. Its most useful property is its straightforward implementation. Moreover, our method allows complete control of the mixing properties of the map, independent of the invariant density.

\section{Acknowledgements}

We thank the referees for their useful comments. This work was supported by the Irish science and technology agency, Enterprise Ireland, under research grant No. SC-00-86 


\section{A Transition matrix - supplementary material}

The special form of the matrix $A$ arises in the context of communication networks, and its origins are described thoroughly in [13] and [14]. Essentially, the matrix comes about from a model of synchronised information sources operating an Additive-Increase Multiplicative-Decrease (AIMD) congestion control algorithm. Networks of such devices in the presence of a bottleneck buffer may be modelled as a positive linear system, whence we get the $A$ matrix. More recent results will be found in [16]. We present some further properties of the matrix $A$ below, which are proved in the aforementioned papers.

Lemma 1 For a positive column stochastic matrix $A$ of the following form: $\operatorname{diag}\left(\beta_{1}, \ldots, \beta_{n}\right)+\left(1 / \sum_{i=1}^{n} \alpha_{i}\right)\left[\alpha_{1}, \ldots, \alpha_{n}\right]^{T}\left[1-\beta_{1}, \ldots, 1-\beta_{n}\right]$, and $\beta_{i} \in(0,1)$, $\alpha_{i}>0$, then the dominant (Perron) eigenvector of $A$, corresponding to the sole unity eigenvalue is given by

$$
x_{p}=\theta\left[\frac{\alpha_{1}}{1-\beta_{1}}, \ldots, \frac{\alpha_{n}}{1-\beta_{n}}\right], \theta \in R
$$

Theorem 1 Consider the matrix A in Lemma 1. The following statements are true:

(1) Except for the Perron eigenvalue, all of the eigenvalues lie in the interval $\left[\beta_{1}, \beta_{n}\right]$.

(2) If all the $\beta$ s are distinct, then $\beta_{1}<\lambda_{1}<\beta_{2}<\ldots<\beta_{n}<\lambda_{n}=1$

Outline Proof: The matrix $A$ is diagonally similar to a matrix of the form $G^{-1}\left(D+\alpha \beta^{T}\right) G$ where $D=\operatorname{diag}\left(\beta_{1}, \ldots, \beta_{n}\right)$, and $G$ is a diagonal matrix (simple calculation). Using Lemma 1 together with this result, and standard results on the symmetric eigenvalue problem (see for instance Theorem 8.6.2 in [17], or [18]), the proof of (1) and (2) follow directly.

\section{References}

[1] S. Ulam, J. von Neumann, On combinations of stochastic and deterministic processes, Bulletin of the American Mathematical Society 53 (1947) 1120.

[2] R. L. Kautz, Using chaos to generate white noise, Journal of Applied Physics 86 (10) (1999) 5794-5800.

[3] L. Kocarev, Chaos-based cryptography: A brief overview, IEEE Circuits and Systems Magazine 1 (3) (2001) 6-21.

[4] M. Delgado-Restituto, A. Rodriguez-Vazquez, Integrated chaos generators, Proceedings of the IEEE 90 (5) (2002) 747-767. 
[5] A. Boyarsky, P. Góra, Chaotic maps derived from trajectory data, Chaos 12 (1) (2002) 42-48.

[6] H. G. Schuster, Deterministic Chaos, VCH, 1989.

[7] E. Ott, Chaos in Dynamical Systems, 2nd Edition, Cambridge University Press, 2002 .

[8] A. Lasota, M. Mackey, Chaos, Fractals, and Noise, Vol. 97 of Applied Mathematical Sciences, Springer-Verlag, 1994.

[9] S. M. Ulam, A Collection of Mathematical Problems, Vol. 8 of Interscience Tracts in Pure and Applied Math, Interscience, 1960.

[10] T. Li, Finite approximation for the frobenius-perron operator: A solution to ulam's conjecture, Journal of Approximation Theory 17 (1976) 177-186.

[11] E. M. Bollt, Controlling chaos and the inverse frobenius-perron problem: Global stabilization of arbitrary invariant measures, International Journal of Bifurcation and Chaos 10 (5) (2000) 1033-1050.

[12] P. Góra, A. Boyarsky, A matrix solution to the inverse frobenius-perron problem, Proceedings of the American Mathematical Society 118 (2) (1993) 409-414.

[13] R. Shorten, D. Leith, J. Foy, R. Kilduff, Analysis and design of synchronised communication networks, in: Proceedings of 12th Yale Workshop on Adaptive and Learning Systems, 2003.

[14] A. Berman, R. Shorten, D. Leith, Positive matrices associated with synchronised communication networks, Linear Algebra and Its Applications (Accepted).

[15] D. G. Luenberger, Introduction to Dynamic Systems, Wiley, 1979.

[16] D. Leith, et al., Stochastic equilibria of aimd communication networks, submitted to SIAM Journal on Matrix Analysis and Applications.

[17] G. Golub, C. van Loan, Matrix Computations, Johns Hopkins University Press, 1996.

[18] R. Horn, C. Johnson, Matrix Analysis, Cambridge University Press, 1985. 